\title{
NOTE ON SINGULAR INTEGRALS ${ }^{1}$
}

E. M. STEIN

1. Statement of result. ${ }^{2}$ Let

$$
\tilde{f}(x)=(\mathrm{P} . \mathrm{V} \cdot / \pi) \int_{-\infty}^{+\infty}[f(y) d y] /(x-y) ;
$$

then by a well-known theorem of M. Riesz,

$$
\|\tilde{f}(x)\|_{p} \leq A_{p}\|f(x)\|_{p}, \quad \text { if } 1<p<\infty .
$$

Hardy and Littlewood [4], and Babenko [1], have complemented this result by proving that:

$$
\|\tilde{f}(x)|x| \beta\|_{p} \leq A_{p, \beta}\|f(x)|x| \beta\|_{p},
$$

if $1<p<\infty$, and $-1 / p<\beta<1 / p^{\prime}$.

The theory of conjugate functions has been extended to $n$-dimensions by A. P. Calderón and A. Zygmund. In [2] and [3] they have considered a wide variety of singular transformations of the form:

$$
T(f)(x)=\text { P.V. } \int_{E_{n}}\left\{[H(x, x-y)] /|x-y|^{n}\right\} f(y) d y,
$$

and have proved that under suitable conditions on $H$,

$$
\|T f\|_{p} \leq A_{p}\|f\|_{p}, \quad 1<p<\infty .
$$

Our aim is to prove the following:

Theorem. Let $(T f)(x)=P . V . \int_{E_{n}}\left\{[H(x, x-y)] /|x-y|^{n}\right\} f(y) d y$, and assume that $\|T(f)(x)\|_{p} \leq A_{p}\|f(x)\|_{p}, 1<p<\infty$. Assume further that $|H(x, \quad x-y)| \leq A$. Then $\|(T f)(x)|x| \beta\|_{p} \leq A_{p, \beta}\left\|f(x)|x|^{\beta}\right\|_{p}$ if $1<p<\infty$, and $-n / p<\beta<n / p^{\prime}$.

The proof will depend mainly on the following lemma.

Lemma. Let

$$
K(x, y)=\left|1-(|x| /|y|)^{\beta}\right| /|x-y|^{n},
$$

and let

Received by the editors May 7, 1956.

1 The research was done while the author was a National Science Foundation Fellow.

2 The problem considered here was suggested by Professor A. Zygmund. 


$$
U(f)(x)=\int_{E_{n}} K(x, y) f(y) d y .
$$

Then

$$
\|U(f)(x)\|_{p} \leq A_{p, \beta}\|f(x)\|_{p}, \quad \text { if }-n / p<\beta<n / p^{\prime} .
$$

This lemma is well-known if $n=1$, but the proof in the general case is more difficult.

2. Proof of the lemma. Given a vector $x=\left(x_{1}, x_{2}, \cdots, x_{n}\right)$, let $\xi$ denote the unit vector whose direction is that of $x$, and $|x|=r$. Similarly let $\eta$ denote the unit vector whose direction is $y$ and $|y|=R$; that is, $x=r \xi$, and $y=R \eta$. Let $d \omega_{\xi}$, and $d \omega_{\eta}$ respectively denote the elements of Euclidean measure on the spheres $|x|=1,|y|=1$, and let $\Sigma$, and $\Sigma^{\prime}$ denote these respective unit spheres. In what follows, $A$ will denote a general constant.

Let us set $\lambda=|y| /|x|$. We then notice that the kernel $K(x, y)$ offers three difficulties: near $\lambda=0, \lambda=1$, and $\lambda=\infty$. For this reason we break up consideration into these cases. In fact, let $K_{1}(x, y)$ $=K(x, y)$ if $0 \leq \lambda \leq 1 / 2$, zero otherwise; $K_{2}(x, y)=K(x, y)$ if $2 \leq \lambda$, zero otherwise; and $K_{3}(x, y)=K(x, y)$ if $1 / 2<\lambda<2$, zero otherwise.

Assume, without loss, that $f(x) \geq 0$, and denote by

$$
T_{i}(f)=\int_{E_{n}} K_{i}(x, y) f(y) d y, \quad i=1,2,3 .
$$

Let us consider $T_{1}$ first. Then

$$
T_{1}(f)(r \xi)=\int_{\Sigma^{\prime}} \int K_{1}(r \xi, R \eta) f(R \eta) R^{n-1} d R d \omega_{\eta}
$$

now make the change of variables $R=\lambda r$, and (1) becomes:

$$
T_{1}(f)(r \xi)=\int_{\Sigma^{\prime}} \int_{0}^{1 / 2} K_{1}(\xi, \lambda \eta) f(\lambda r \eta) \lambda^{n-1} d \lambda d \omega_{\eta}
$$

owing to the homogeneity of order $-n$ of $K_{1}(x, y)$. But we have

$$
K_{1}(\xi, \lambda \eta)=\left[\left|1-\lambda^{-\beta}\right|\right] /\left[1-2 \lambda \cos (\xi, \eta)+\lambda^{2}\right]^{n / 2},
$$

where $(\xi, \eta)$ indicates the angle between the vectors $\xi$ and $\eta$. While, $\left[1-2 \lambda \cos (\xi, \eta)+\lambda^{2}\right]^{n / 2} \geq A>0$, if $0 \leq \lambda \leq 1 / 2$. Therefore

$$
\left|T_{1}(f)(r \xi)\right| \leq A \int_{\Sigma^{\prime}} \int_{0}^{1 / 2}\left|1-\lambda^{-\beta}\right| \lambda^{n-1} f(\lambda r \eta) d \lambda d \omega_{\eta} .
$$

Now define $F_{\xi}(r)$ by 


$$
F_{\eta}(r)=\int_{0}^{1 / 2}\left|1-\lambda^{-\beta}\right| \lambda^{n-1} f(\lambda r \eta) d \lambda
$$

and we note that

$$
\left[\int_{0}^{\infty}|g(\lambda r)|{ }^{p} \boldsymbol{r}^{n-1} d r\right]^{1 / p}=\lambda^{-n / p} \int_{0}^{\infty}|g(\boldsymbol{r})|^{p^{n-1}} d \boldsymbol{r} .
$$

Hence applying (5) to (4), via Minkowski's inequality for integrals, gives:

$$
\begin{aligned}
& {\left[\int_{0}^{\infty}\left|F_{\eta}(\boldsymbol{r})\right|{ }^{{ }^{n} \boldsymbol{r}^{n-1}} d r\right]^{1 / p}} \\
& \quad \leq\left[\int_{0}^{1 / 2}\left|1-\lambda^{-\beta}\right| \cdot \lambda^{n-1} \cdot \lambda^{-n / p} d \lambda\right] \cdot\left[\int_{0}^{\infty}|f(\boldsymbol{r} \eta)|^{p} \boldsymbol{r}^{n-1} d \boldsymbol{r}\right]^{1 / p} .
\end{aligned}
$$

But $\int_{0}^{1 / 2}\left|1-\lambda^{-\beta}\right| \lambda^{n-1} \cdot \lambda^{-n / p} d \lambda<\infty$ if $\beta<n / p^{\prime}$. Hence (6) yields

$$
\left[\int_{0}^{\infty}\left|F_{\eta}(r)\right|{ }^{p}{ }^{n-1} d r\right]^{1 / p} \leq A\left[\int_{0}^{\infty}|f(r \eta)|{ }^{p} r^{n-1} d r\right]^{1 / p} .
$$

Now

$$
\begin{aligned}
& \int\left[\int F_{\eta}(\boldsymbol{r}) d \omega_{\eta}\right]^{p} \boldsymbol{r}^{n-1} d r \leq A \iint\left[F_{\eta}(\boldsymbol{r})\right]^{p} d \omega_{\eta} \boldsymbol{r}^{n-1} d \boldsymbol{r} \\
= & A \int d \omega_{\eta} \int\left[F_{\eta}(\boldsymbol{r})\right]^{p} \boldsymbol{r}^{n-1} d r \leq A \int d \omega_{\eta} \int[f(\eta \boldsymbol{r})]^{p^{n}} \boldsymbol{r}^{n-1} d \boldsymbol{r}=A\|f\|_{\boldsymbol{p}} .
\end{aligned}
$$

Here we have made use of (7) and the fact that the total measure of $d \omega_{\eta}$ is finite. However by (3)

$$
\left|T_{1}(f)(r \xi)\right| \leqq \int_{\Sigma^{\prime}} F_{\eta}(r) d \omega_{\eta}
$$

Therefore (8) implies that

$$
\left\|\left(T_{1}\right)(f)(r \xi)\right\|_{p} \leq\left\|\int F_{\eta}(r) d \omega_{\eta}\right\|_{p} \leq A\|f\|_{p} .
$$

The $L_{p}$ boundedness is therefore proved for $T_{1}(f)$. The case where $|y| /|x|=\lambda \geq 2$ is treated in the same manner. We get

$K_{2}(r \xi, R \eta)=\left|1-\lambda^{-\beta}\right| /\left[1-2 \lambda \cos (\xi, \eta)+\lambda^{2}\right]^{n / 2} \leqq A\left|1-\lambda^{-\beta}\right| / \lambda^{n}$.

Since $\lambda \geq 2$, matters now depend on the convergence of the integral 


$$
\int_{2}^{\infty}\left|1-\lambda^{-\beta}\right|\left[\lambda^{n-1} / \lambda^{n}\right] \cdot \lambda^{-n / p} d \lambda
$$

which clearly converges if $\beta>-n / p$.

We now proceed to the case where $1 / 2<\lambda<2$, and in the previous notation we have:

$$
\begin{aligned}
& T_{3}(f)(r \xi) \\
& \quad=\int_{\Sigma^{\prime}} d \omega_{\eta} \int_{1 / 2}^{2}\left\{\left[\left|1-\lambda^{-\beta}\right| \cdot \lambda^{n-1}\right] /\left[1-2 \lambda \cos (\xi, \eta)+\lambda^{2}\right]^{n / 2}\right\} \\
& \quad \cdot f(\lambda r \eta) d \lambda
\end{aligned}
$$

and write $G_{\lambda}(r \xi)$ for the following:

$$
\begin{aligned}
& G_{\lambda}(r \xi) \\
& \quad=\int_{\Sigma^{\prime}} f(\lambda r \eta)\left\{\left[1-\lambda^{-\beta} \mid \cdot \lambda^{n-1}\right] /\left[1-2 \lambda \cos (\xi, \eta)+\lambda^{2}\right]^{n / 2}\right\} d \omega_{\eta} .
\end{aligned}
$$

Now observe that if $1 / 2<\lambda<2$, the expression within the brackets is bounded up to a constant multiple by Poisson's Kernel for the $(n-1)$ sphere. Using a well known property of the Poisson Kernel, we get from (10)

$$
\int_{\Sigma}\left|G_{\lambda}(r \xi)\right|^{p} d \omega_{\xi} \leq A \int_{\Sigma^{\prime}}|f(\lambda r \eta)|^{p} d \omega_{\eta}, \quad \text { if } 1 / 2<\lambda<2 .
$$

Now integrate (11) with respect to $r^{n-1} d r$; this gives

$$
\int_{0}^{\infty} \int_{\Sigma}\left|G_{\lambda}(r \xi)\right| p d \omega_{\xi} \eta^{n-1} d r \leq A \int_{0}^{\infty} \int_{\Sigma^{\prime}}|f(\lambda r \eta)|^{p} d \omega_{\eta} r^{n-1} d r .
$$

However $\|f(\lambda x)\|_{p}=\lambda^{-n / p}\|f(x)\|_{p}$. Therefore (12) yields

$$
\left\|G_{\lambda}\right\|_{p} \leq A \lambda^{-n / p}\|f\|_{p}
$$

However,

$$
T_{3}(f)=\int_{1 / 2}^{2} G_{\lambda} d \lambda .
$$

Therefore, Minkowski's inequality for integrals applied to (13) and (14) gives

$$
\left\|T_{3}(f)\right\|_{p}=\left\|\int_{1 / 2}^{2} G_{\eta} d \lambda\right\| \leq \int_{1 / 2}^{2}\left\|G_{\lambda}\right\|_{p} d \lambda \leq A\|f\|_{p}
$$

and the proof of the lemma is complete. 
3. Proof of the theorem. Let now

$$
T\left(f^{\prime}\right)=\text { P.V. } \int_{E_{n}}\left\{[H(x, x-y)] /|x-y|^{n}\right\} f(y) d y,
$$

where $T$ satisfies the conditions of the theorem. Write $F(x)=T[f(y)]$, and $F^{*}(x)=T\left[|y|^{\beta} f(y)\right]$. Then by the assumptions of the theorem:

$$
\left\|F^{*}(x)\right\|_{p} \leq A_{p}\left\||x|^{\beta} f(x)\right\|_{p} .
$$

It is therefore sufficient to prove that:

$$
\left\|F^{*}(x)-|x| \beta F(x)\right\|_{p} \leq A_{p, \beta}\left\||x|^{\beta} f(x)\right\|_{p} .
$$

However,

$$
\begin{aligned}
& \left.\left|F^{*}(x)-\right| x\right|^{\beta} F(x) \mid \\
& \quad=\left|\int_{E_{n}}\left\{[H(x, x-y)] /|x-y|^{n}\right\}\left[|y|^{\beta}-|x|^{\beta}\right] f(y) d y\right| \\
& \quad=\left.\left|\int_{E_{n}}\left\{[H(x, x-y)] /|x-y|^{n}\right\}\left[1-\left(|x|^{\beta} /|y|^{\beta}\right)\right]\right| y\right|^{\beta} f(y) d y \mid \\
& \quad \leq A \int_{E_{n}} K(x, y)|y|^{\beta}|f(y)| d y,
\end{aligned}
$$

by the assumption that $|H(x, x-y)| \leq A$.

Hence we need only appeal to the lemma to complete the proof.

\section{REFERENCES}

1. K. I. Babenko, On conjugate functions, Doklady Akademii Nauk SSSR. (N.S.) vol. 62 (1948) pp. 157-160 (Russian).

2. A. P. Calderon and A. Zygmund, On the existence of certain singular integrals, Acta Math. vol. 88 (1952) pp. 85-139.

3. - On singular integrals, Amer. J. Math. vol. 78 (1956) pp. 289-309.

4. G. H. Hardy and J. E. Littlewood, Some more theorems concerning Fourier series and Fourier power series, Duke Math. J. vol. 2 (1936) pp. 354-382.

University of Chicago 